\title{
String Bowing Gestures at Varying Bow Stroke Frequencies: A Case Study
}

\author{
Nicolas Rasamimanana ${ }^{1}$, Delphine Bernardin ${ }^{2}$, Marcelo Wanderley ${ }^{2}$, and \\ Frédéric Bevilacqua $^{1}$ \\ 1 IRCAM, CNRS - UMR 9912, 1 Place Igor Stravinsky, 75004 PARIS, France \\ \{Nicolas.Rasamimanana, Frederic.Bevilacqua\}@ircam.fr \\ 2 IDMIL/CIRMMT, McGill University, Montreal, QC, Canada \\ \{Delphine.Bernardin, Marcelo.Wanderley\}@mcgill.ca
}

Introduction: The understanding of different bowing strategies can provide key concepts for the modelling of music performance. Such model can be applied in music pedagogy or in the design of novel musical interfaces.

We previously reported the study of three standard bowing techniques in violin playing [1]. In particular, we discussed issues on gesture "continuity". This concept relates to the fact that an expert violinist is able to play several subtle variations between two bowing techniques, e.g. Détaché and Martelé. We showed in [1] that such a wide range of variation can be directly tracked in the bowing dynamics and described with features derived from bow acceleration profiles.

In this paper, we elaborate on this topic of gesture continuity in violin playing. Slow bowing generally requires a relaxed right arm. Nevertheless, it is usually recognized among bowed string players that rapid, repeated bow strokes can require the right arm to be tensed up. Such strategy is usually used to achieve a given rhythm, or to perform tremolos. Hence, from the players' viewpoint, there are different, possible arm movements to perform cyclic, repetitive bowings. We here test this hypothesis with the study of an accelerando/decelerando, i.e. bow strokes performed with an increasing/decreasing frequency.

Related works: We hypothesize that players can change their bowing gestures to perform high bowing frequencies. Several studies in motor control dealt with the reorganisation of coordinative patterns, e.g. gait shifting. In particular, Kelso studied the abrupt phase transitions in human hand movements according to the cycling frequency [2]: when increasing the frequency of an out-of-phase motion, human's finger movements shift to an in-phase motion. Winold et al. [3] first studied coordination issues in cellists' bow and arm movements. They concluded that increasing tempi produced a proportional scaling of strokes amplitudes and durations and did not notice any change in within-limb coordinations.

Method: We recorded the movements, bow pressure on strings and audio from two advanced violin and viola players from McGill Schulich School of Music. They performed an accelerando from medium paced Détaché to a tremolo "as 
fast as possible" tied with a decelerando back to medium paced Détaché on one fixed note. Movements were recorded with a Vicon System 460 optical tracker, and bow pressure with a custom sensor designed at Ircam.

Results and Discussion: The analysis of bow motion showed the existence of two types of bow velocity and acceleration profiles, therefore defining two bowing gestures: for lower bow stroke frequencies, bow velocity is close to a square shape, for higher frequencies it shifts to a smooth, almost sinusoid shape (Figure 1 left).

The arm joint angle profiles follow a similar change. Moreover, arm joint angles also indicates the possible existence of a later, within-limb change of coordination. In the case of the violin player, the elbow and the wrist first start in out-of-phase and shift to in-phase to achieve the fastest part of the accelerando/decelerando (Figure 1 right).

Interestingly, the effect on sound is more subtle. A spectrum analysis does not reveal any clear concomitant change to the dramatic change in the bow velocity profile. We can argue that this similarity in sound is intended by instrumentalists, adjusting unconsciously other parameters, so that the abrupt change in bowing gesture is not hearable. However, we may also hypothesize that this change in bowing gesture has still an effect on finer timbre aspects (e.g. transitions between notes). This exploratory study is currently completed with the measurement and analysis of more players to validate the hypothesis of a change in arm coordination.
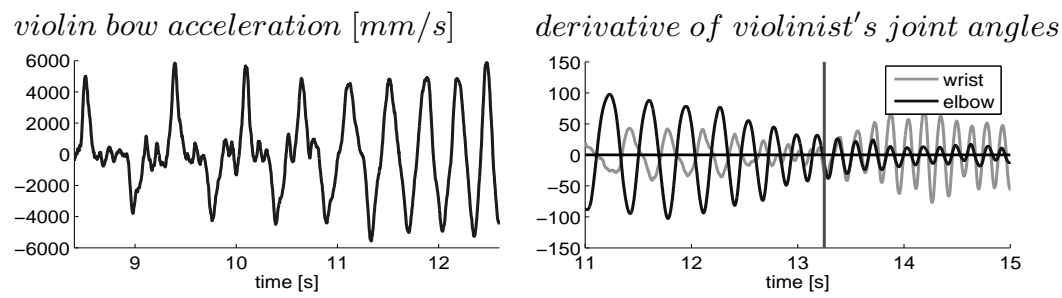

Fig. 1. Left: Change in bow acceleration. Right: Possible change in limb coordination.

\section{References}

1. Rasamimanana, N.H., Flety, E., Bevilacqua, F.: Gesture analysis of violin bow strokes. In Gibet, S., Courty, N., Kamp, J.F., eds.: Lecture Notes in Artificial Intelligence, LNAI 3881, Springer Verlag (2006) 145-155

2. Haken, H., Kelso, J.A.S., Bunz, H.: A theoretical model of phase transitions in human hand movements. Biological cybernetics 51(5) (1985) 347-356

3. Winold, H., Thelen, E., Ulrich, B.D.: Coordination and control in the bow arm movements of highly skilled cellists. Ecological Psychology 6(1) (1994) 1-31 